\title{
Geociências
}

\section{Estudos por $\mu$ XRF-Sincrotron de fluidos mineralizadores detectados em minérios uraníferos, ferríferos e depósitos epitermais}

\author{
Francisco Javier Rios \\ CDTN/CNEN, Belo Horizonte, Brasil.E-mail: javier@cdtn.br \\ Kazuo Fuzikawa \\ CDTN/CNEN,Belo Horizonte, Brasil.E-mail:kazuo@cdtn.br \\ James Vieira Alves \\ CDTN/CNEN, Belo Horizonte, Brasil.E-mail: alvesjv@cdtn.br \\ Rhaine Matos Gonçalves \\ CDTN/CNEN, Belo Horizonte, Brasil.E-mail: pgcdtn@cdtn.br \\ Alexandre de Oliveira Chaves \\ CDTN/CNEN, Belo Horizonte, Brasil.E-mail: aoc@cdtn.br \\ André Moreira de Souza \\ CDTN/CNEN, Belo Horizonte, Brasil.E-mail:pgcdtn@cdtn.br \\ Adriana Mônica Dalla Vecchia Chaves \\ CDTN/CNEN, Belo Horizonte, Brasil. E-mail: pgcdtn@cdtn.br
}

\section{Resumo}

O método de análise não-destrutivo denominado Microfluorescência de Raios X. ( $\mu$ SXRF, radiação Sincrotron) tem sido utilizado recentemente em laboratórios de luz Sincrotron de vários países para identificar e, em situações especiais, quantificar elementos menores, maiores e traços em inclusões fluidas. Com esse intuito, a estação de $\mu$ SXRF do LNLS (Laboratório Nacional de Luz Sincrotron, Campinas) foi utilizada para desenvolver estudos composicionais de inclusões fluidas em minerais transparentes (quartzo, adulária, esmeralda, piroxênio) de diferentes jazidas minerais e minérios opacos de $\mathrm{Ag}$ e Fe.

Palavras-chave: Luz Sincrotron ( $\mu \mathrm{SXRF})$, inclusões fluidas, minérios.

\author{
Ana Rosa Passos Pereira \\ CDTN/CNEN, Belo Horizonte, Brasil,E-mail: arpp@cdtn.br \\ Tatiana Aparecida Fernandes de Lima \\ CDTN/CNEN, Belo Horizonte, Brasil.E-mail: tafl@cdtn.br \\ José Marques Correia Neves \\ CDTN/CNEN,Belo Horizonte, Brasil.E-mail:nevesj@cdtn.br
}

Carlos Alberto Pérez

LNLS, Laboratório Nacional de Luz Sincrotron, Campinas, Brasil.E-Mail:perez@Inls.br

Maurício Gonzalez Guillot

INREMI-UNLP, La Plata, Argentina. E-mail: g_guillot@cadic.gov.ar

Evando Carelle Mattos

INB/URA-Caetité, Bahia, Brasil. E-mail: carelle@inb.br

Carlos Alberto Rosiére

IGC-UFMG, Belo Horizonte, Brasil.E-mail: crosiere@gmail.com

\begin{abstract}
Synchrotron radiation micro $X$-ray fluorescence

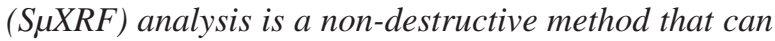
be used to identify, and in special cases, to quantify major, minor, and trace elements present in fluid inclusions. In spite of the relevant results, this technique has been used only in a few studies, which, moreover, are mostly focused on either synthetic or natural fluid inclusions in transparent minerals. To investigate fluid inclusions in transparent and opaque minerals, the synchrotron radiation $X$-ray microprobe station from the $\mu S X R F$ fluorescence beamline at the LNLSCampinas was used. This analytical method has been proven to be efficient in the chemical characterization of the fluid inclusions constituents of some transparent (quartz, adularia, emerald, etc.) and opaque minerals (pyrargyrite and hematite).
\end{abstract}

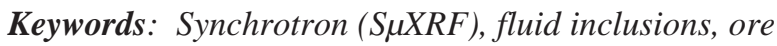
minerals. 


\section{Introdução}

A luz, ou radiação, Sincrotron é uma designação ampla para todos os tipos de luz originados por partículas relativísticas em trajetórias curvas. Compreende uma faixa de luz que vai do ultravioleta até os raios $\mathrm{X}$ moles, atingindo, em determinadas circunstâncias, os raios X duros. As radiações Sincrotron são produzidas em um equipamento que acelera partículas (elétrons) a grandes velocidades, emitindo fótons (grãos de luz). Portanto a radiação Sincrotron é uma radiação eletromagnética produzida quando os elétrons (ou pósitrons), utilizando magnetos, são forçados a mudar de direção. A radiação é um milhão de vezes mais brilhante do que as fontes convencionais e, em termos de magnitude, 11 vezes mais brilhante que a produzida pelos raios X normais. Quando o feixe de raios $\mathrm{X}$ irradia a amostra, origina uma interação com os constituintes atômicos da mesma a partir de três processos principais: o efeito fotoelétrico, o efeito de dispersão elástica (Rayleigh) e o efeito inelástico (Winick \& Doniach, 1980; Lindgreen, 2000).

Desde a sua descoberta, em 1940, a Luz Sincrotron tem-se convertido numa excelente fonte de raios X, tendo, atualmente, grandes aplicações científicas em muitas disciplinas, incluindo as seguintes: Biologia, Medicina, Física, Engenharias e Geociências. Atualmente, os equipamentos dos aceleradores de partículas Sincrotron já se encontram em sua terceira geração.

Em geociências, a Luz Sincrotron pode ser utilizada no estudo das inclusões fluidas presentes nos minerais, através da técnica denominada Microfluorescência de raio $X(\mu \mathrm{SXRF})$. Com esse objetivo, uma microssonda de raios $\mathrm{X}$ é utilizada para atingir uma resolução espacial micrométrica. Os primeiros estudos desse tipo foram desenvolvidos por Frantz et al. (1988), em inclusões fluidas sintéticas. Entretanto a maior parte dos estudos de inclusões fluidas, em minerais transparentes, foram desenvolvidos recentemente por Rankin et al. (1992), Mavrogenes et al., (1995), Philippot et al., (1998 e 2001), Vanko e Mavrogenes (1998); Hayashi e Lida (2001), Vanko et al., (2001); Menez et al., (2002) e Samson et al., (2003). No Brasil, os primeiros estudos de inclusões fluidas em minerais transparentes foram desenvolvidos por Xavier et al., (1999), Alves et al., (2000a, b) e Rios et al. (2006), na Estação de Microfluorescência de Raios X Sincrotron ( $\mu \mathrm{SXRF}$ ), instalada no Laboratório Nacional de Luz Sincrotron (LNLS) em Campinas, SP.

O LNLS constitui-se no único laboratório desse tipo disponível na América do Sul. A estação está equipada com um capilar cônico ultrafino, que condensa o feixe de luz branca de $0,5 \mathrm{~mm}$ por $0,5 \mathrm{~mm}$ numa área de, aproximadamente, $20 \mu \mathrm{m}$ (Perez et al., 1999). Na linha da $\mu$ SXRF do LNLS, é utilizado um feixe branco ou monocromático. Também fazem parte da mesma uma janela de berilo, para isolar o vácuo da linha, um cristal monocromador channel-cut e um conjunto de fendas motorizadas controladas por computador, para limitar o feixe de luz. Detetores por dispersão de energia $\mathrm{Si}(\mathrm{LI})$ e HPGe integram o sistema de detecção da linha de luz (Perez et al. 1999) .

Com a $\mu \mathrm{SXRF}$, as radiações características dos elementos mais pesados são menos absorvidas, se comparadas com as dos elementos mais leves. O método é não-destrutivo e permite, em casos especiais, quantificar elementos maiores, menores e traços contidos nas inclusões fluidas. Trabalhando em condições normais de pressão e temperatura, é possível detectar elementos com números atômicos maiores que o alumínio (Vanko \& Mavrogenes, 1998). Portanto, o Na (um dos principais constituintes das inclusões fluidas) não pode ser detectado por essa técnica.

A metodologia da $\mu \mathrm{SXRF}$ fornece resultados semiquantitativos de forma rápida. A análise dos dados de inclusões fluidas, utilizando a $\mu \mathrm{SXRF}$, inclui a comparação do espectro da inclusão com respeito ao espectro do mineral hospedeiro (denominado espectro branco) e a identificação dos picos de difração de cada elemento (Figura 1).

O presente trabalho mostra os resultados obtidos dos estudos realizados em minerais transparentes (piroxênios, feldspatos, entre os quais a adulária, esmeraldas, calcita e quartzo) e, ainda, opacos (hematita e pirargirita), utilizando-se a Estação de $\mu$ SXRF do LNLS de Campinas (Figuras 1 e 2).

\section{Alguns exemplos minerais estudados pela $\mu S X R F$ Quartzo}

Foi o mineral mais estudado, apresentando, geralmente, bons resultados em jazidas de Ag-Au epitermais, rochas graníticas e pegmatíticas. No caso de quartzo de depósitos epitermais do Macizo del Deseado, Patagônia, os resultados da $\mu \mathrm{SXRF}$ (presença de $\mathrm{Cl}, \mathrm{K}$, $\mathrm{Ca}, \mathrm{Fe}$, and $\mathrm{Sb}$ ) são concordantes com os dados microtermométricos, confirmando a presença do sistema salino $\mathrm{H}_{2} \mathrm{O}+\mathrm{NaCl}+\mathrm{KCl}+\mathrm{CaCl}_{2}+\mathrm{FeCl}_{2}$.

\section{Piroxênios}

Os resultados obtidos, nesses minerais, associados à mineralização uranífera da Jazida Cachoeira, Lagoa Real, $\mathrm{BA}$, indicaram a presença de V nas soluções mineralizadoras. Os outros picos encontrados coincidem com os correspondentes ao hospedeiro $(\mathrm{Si}, \mathrm{Ca}, \mathrm{Ti}$, $\mathrm{Mn}, \mathrm{Fe}$ e Zn). Não foi identificado o pico de $\mathrm{U}$, o que confirma observações petrográficas recentes (somente da jazida da Cachoeira), que sugerem ser o piroxênio imediatamente anterior ao principal evento uranífero na região (Chaves et al., 2006).

\section{Esmeraldas}

Foram obtidos excelentes resultados em inclusões maiores de $50 \mu \mathrm{m}$ da 
região de Nova Era (MG) (Figura 2). Os dados semiquantitativos obtidos confirmaram a presença de $\mathrm{Cl}, \mathrm{Ca}, \mathrm{K}, \mathrm{V}, \mathrm{Cr}, \mathrm{Mn}$, $\mathrm{Fe}, \mathrm{Ni}, \mathrm{Cu}, \mathrm{Zn}$ e As no fluido mineralizador que originou essas gemas.

\section{Adulária}

Apesar das limitações ópticas, o estudo da $\mu$ SXRF, em adulárias de ambientes epitermais, evidenciou a presença de $\mathrm{K}^{+}$e, provavelmente, $\mathrm{Ba}$ no fluido mineralizador.

\section{Hematita e Pirargirita}

Os estudos, por $\mu \mathrm{SXRF}$, nesses minerais opacos, foram desenvolvidos utilizando uma câmera que opera no espectro da luz visível e o início do espectro de infravermelho (near infrared) (Rios et al., 2006). Nas hematitas estudadas (provenientes do Quadrilátero Ferrífero$\mathrm{MG})$, foi detectada a presença de $\mathrm{Ca}$ e provável Fe, Ba, Ti e K nas soluções. No caso da pirargirita (sulfeto de Ag e As), foi confirmada a presença de $\mathrm{S}, \mathrm{Cu}$ e As, além de traços de Ag nas soluções presentes nas inclusões fluidas.

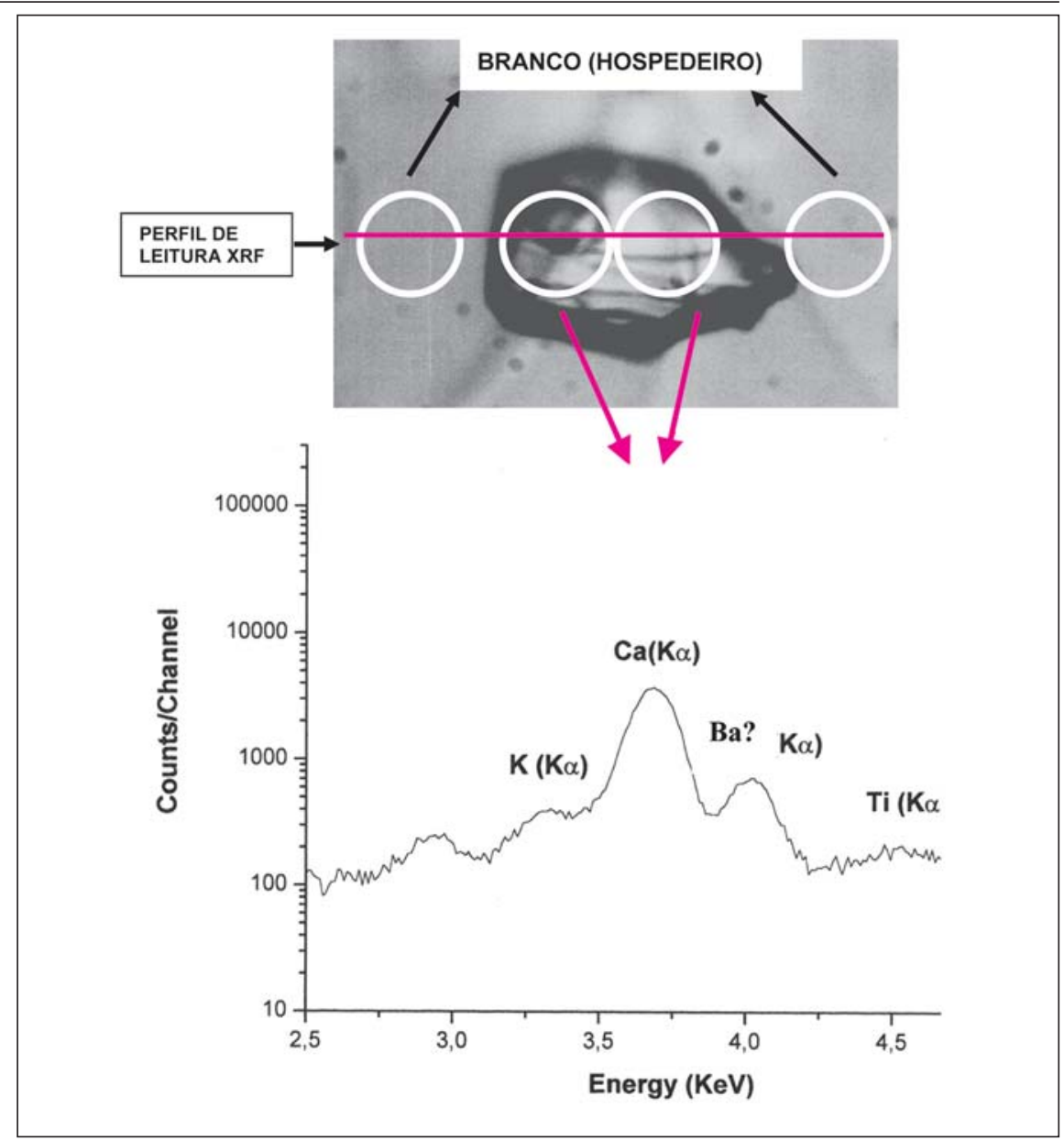

Figura 1 - Procedimento de estudo utilizado durante as análises de inclusões fluidas na Linha $\mu$ SXRF. O feixe é focalizado, inicialmente, no mineral hospedeiro (branco) e, posteriormente, na inclusão fluida. Os dados obtidos são processados com um software específico e, dessa forma, é possível detectar os elementos presentes no mineral hospedeiro e no fluido da inclusão. A fotomicrografia mostra uma inclusão fluida em hematita, analisada na linha XRF.

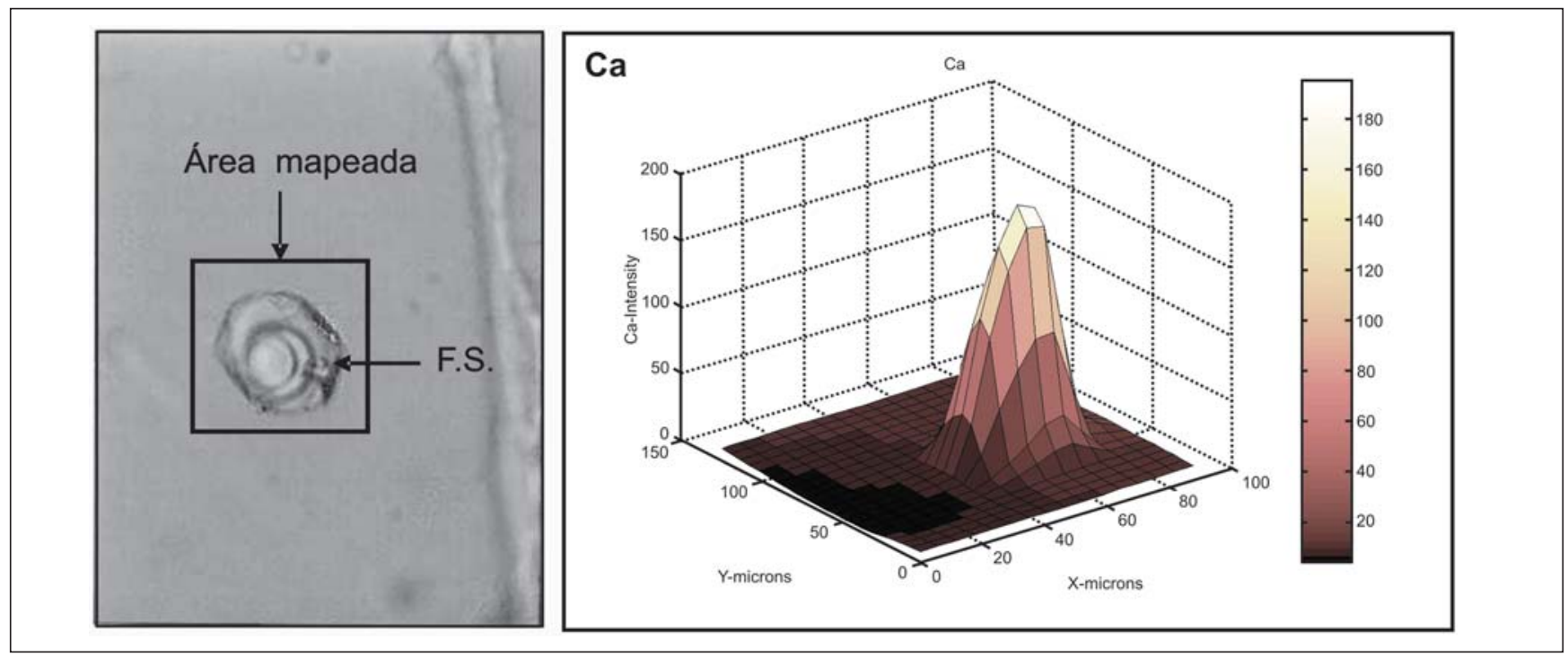

Figura 2 - A fotomicrografia mostra uma inclusão fluida polifásica em esmeralda do garimpo de Capoeirana, Nova Era, MG. O tamanho da inclusão fluida é de $50 \mathrm{~m} \mathrm{~m}$, estando localizada a $14 \mathrm{~m} \mathrm{~m}$ de profundidade em relação à superfície da lâmina. A imagem ao lado da fotomicrografia mostra uma representação gráfica da presença de cálcio numa das fases sólidas (F. S.) presentes na inclusão fluida. 


\section{Vantagens e desvantagens da metodologia da $\mu X R F$ no estudo de inclusões fluidas Vantagens}

(a) Trata-se de um método não-destrutivo, o que permite preservar a amostra para verificações futuras ou outras análises.

(b) Permite desenvolver leituras qualitativas rápidas sobre elementos presentes nas fases gasosas, líquidas e sólidas das inclusões.

(c) Possibilita comparar a composição do hospedeiro e da inclusão numa única corrida de leitura.

(d) Com a ajuda de programas especiais de computador, disponíveis na linha, é possível elaborar diagramas tridimensionais, mostrando a dispersão dos elementos dentro da inclusão

(e) A superposição dos espectros permite diferenciar os elementos que estão presentes na inclusão dos que pertencem ao mineral hospedeiro.

(f) Trata-se de uma metodologia de ponta já disponível no Brasil.

(g) Os projetos, submetidos e aprovados, contam com apoio do LNLS.

\section{Desvantagens}

(a) $\mathrm{O}$ estudo por $\mu \mathrm{SXRF}$ requer inclusões fluidas acima de $30 \mu \mathrm{m}$ localizadas próximas à superfície (< de 10 $\mu \mathrm{m})$. O diâmetro do feixe é de $20 \mu \mathrm{m}$. Portanto o estudo de inclusões menores de $20 \mu \mathrm{m}$, ou localizadas a profundidades maiores de $10 \mu \mathrm{m}$, fornece resultados pouco confiáveis.

(b) A localização das inclusões fluidas é trabalhosa, já que o equipamento óptico da linha proporciona um único aumento sendo que a definição nem sempre é a melhor.

(c) Não permite desenvolver estudos quantitativos, ainda que, em condições ideais, seja possível obter resultados semiquantitativos.

(d) A utilização do software para calcular o ajuste dos dados obtidos é a parte mais demorada do processo, pois se torna indispensável dispor de tempo para identificar os picos de cada elemento.

(e) Não permite, como se referiu anteriormente, identificar o $\mathrm{Na}$, elemento comum nas soluções salinas presentes nas inclusões dos minerais.

(f) Não permite estudar fases nitro-carbônicas.

(g) A metodologia não consegue determinar o estado de valência dos elementos.

\section{Conclusão}

A metodologia da $\mu$ SXRF é uma excelente técnica já disponível no Brasil para realizar microanálises de elementos, presentes nas inclusões fluidas de minerais transparentes, e até de alguns minerais opacos, com número atômico maior que 14. Surge como uma alternativa confiável das metodologias LA-ICP-MS e PIXE, embora não atinja o grau de detecção que fornecem estas últimas metodologias. No estudo de fases sólidas, é uma boa alternativa ao MEV com EDS. Todos os tipos de minerais estudados, pertencentes aos mais variados ambientes, forneceram resultados confiáveis e permitiram complementar e confirmar as informações fornecidas pela microtermometria, sobretudo no que se refere à composição de sistemas salinos. Trata-se, portanto, de um ótimo complemento para os estudos microtermométricos de aplicação rotineira.

\section{Agradecimentos}

Os autores agradecem o suporte financeiro e técnico da FAPEMIG (processos CRA 175-02 e CRA 594-04) e do Laboratório Nacional de Luz Sincrotron (LNLS, processo D09B-XRF-2838) sem os quais essa pesquisa não teria sido viável. Agradecimentos especiais são dirigidos à INB - Indústrias Nucleares do Brasil; à Dra. Rosa Maria S. Bello (USP); aos Drs. Isidoro B. Schalamuck, Raul de Barrio e Pilar Moreira (INREMI, La Plata, Argentina), aos Técnicos Antonino Mathias e Francisco de Assis Rocha (CDTN-CNEN) e aos editores da Revista REM, Dres. Jório Coelho e Hanna Jordt Evangelista.

\section{Referências bibliográficas}

ALVES, J. V., PEREZ, C. A., FUZIKAWA, $\mathrm{K}$., (a). The use of micro-SXRF in fluid inclusion analyses. In: REUNIÃO ANUAL DE USUÁRIOS DO LNLS, 11. Anais... Campinas. Activity Report, Laboratório Nacional de Luz Síncrotron - ABTLus, 2000, p. 13-14.

ALVES, J. V., PEREZ, C. A., FUZIKAWA, K., (b). Fases sólidas em inclusões fluidas das esmeraldas de Capoeirana, Nova Era, MG: determinaçaõ por micro-SXRF. In: SIMPÓSIO BRASILEIRO DE TRATAMENTO E CARACTERIZAÇÃO DE GEMAS, 1. Anais... Ouro Preto: SBG, 2000. p. 16.

CHAVES, A. O., TUBRETT, M., RIOS, F. J., ALVES, J. V., FUZIKAWA, K., CORREIA NEVES, J. M., MATOS, E. C., OLIVEIRA, L. A. R. de, CHAVES, A. M. D. V., GONÇALVES, R. M., PRATES, S. P., ARDISSON, J. D., MACEDO, W. A. de A., MENDONÇA, R., PEREIRA, A. R. P. Magmatic and metamorphic events related to Lagoa Real uraniferous mineralization (BA-Brazil) and the importance of magmatic arcs in Uranium prospecting. In: CONGRESSO BRASILEIRO DE GEOLOGIA, 43. Anais... Aracaju: SBG, 2006. CD-ROM.

FRANTZ, J. D., MAO, H. K., ZHANG, T, G., WU, Y., THOMPSON, A.C., UNDERWOOD, J. H., GIAUQUE, R. D., JONES, K.W., RIVERS, M. L. Analysis of fluid inclusions by X-ray fluorescence using synchrotron radiation: Chemical Geology, v. 69, p. 235-244, 1988.

GONZALEZ GUILLOT, M., DE BARRIO, R.E., GANEM, F. Mina Martha, yacimiento argentífero Bacon, Macizo del Deseado, Provincia de Santa Cruz. In: MINMET, 7. Anais... Rio Cuarto: Argentina, 2004. p. 205-210. 
Francisco Javier Rios et al.

HAYASHI, K-I, LIDA, A. Preliminary study on the chemical mapping of individual fluid inclusion by synchrotron $\mathrm{x}$-ray fluorescence microprobe. Resource Geology, 51, p. 259-262, 2001

LINDGREEN, E.S. X-Ray fluorescence analysis: energy dispersive. In: MEYERS, R.A. (ed). Enciclopedia of Analytical Chemistry. Chichester: John Wiley \& Sons, 2000. cap. 1, 1-12.

MAVROGENES J.A., BODNAR, R.J., ANDERSON, A. J., BAJT, S., SUTTON, S.R., RIVERS, M.L. Assessment of the uncertainties and limitations of quantitative elemental analysis of individual fluid inclusions using synchrotron X-ray fluorescence (SXRF). Geochim. Cosmochim. Acta, v.59, n. 9, p. 3987-3995, 1995.

MENEZ, B., PHILIPPOT, P., BONNINMOSBAH, M., SIMINOVICI, A., GIBERT, F. Analysis of individual fluid inclusions using synchrotron X-ray fluorescence microprobe: progress toward calibration for trace elements. Geochim. Cosmochim. Acta, v. 66, p.561-576, 2002.

PÉREZ, C.A., RADTKE, M., SÁNCHEZ, J.M., TOLENTINO, H.C.N., NEUENSHWANDER, R.T., BARG, W., RUBIO, M., BUENO, M.I.S., RAIMUNDO, I.M., ROHWEDDER, J. J. R. Synchrotron radiation $\mathrm{x}$-ray fluorescence at the LNLS: Beamline Instrumentation and Experiments. $\boldsymbol{X}$-Ray Spectrometry, v. 28, p. 320-326, 1999.
PHILIPPOT, P., MÉNEZ, B., CHEVALLIER, P., GIBERT, F., LEGRAND, F. AND POPULUS, P. Absorption correction procedures for quantitative analysis of fluid inclusion using synchrotron radiation $\mathrm{X}$-ray fluorescence. Chemical Geology , v. 144, p. 121-136. 1998

PHILIPPOT, P., MÉNEZ, B., DRAKOPOULOS, M., SIMIONOVICI, A., SNIGIREV, A., SNIGIREVA, I. Mapping trace-metal $(\mathrm{Cu}, \mathrm{Zn}, \mathrm{As})$ distribution in a single fluid inclusion using a third generation synchrotron light source. Chemical Geology, v. 173, p.151-158, 2001.

RANKIN, A.H., RAMSEY, M.H., COLES, B., VAN LANGEVELDE, F., THOMAS, C. R. The composition of hypersaline, iron-rich granitic fluids based on laser-ICP and Synchrotron-XRF microprobe analysis of individual fluid inclusions in topaz, Mole, Eastern Australia. Geochim. Cosmochim. Acta, v. 56, p. 67-79, 1992.

RIOS, F. J., ALVES, J.V., PÉREZ, A.C., COSTA, È. C., ROSIÈRE, C.A., FUZIKAWA, K., CORREIA NEVES, J. M., CHAVES, A.O., PRATES, S.P., BARRIO, R.E. Combined investigations of fluid inclusions in opaque ore minerals by NIR/SWIR microscopy and microthermometry and synchrontron radiation X-ray fluorescence. Applied Geochemistry, Special Issue "Frontiers in Analytical Geochemistry", v. 21, n. 5, p. 813-819, 2006.
SAMSON, I., ANDERSON, A., MARSHALL, D. Fluid inclusions: analysis and interpretation. Vancouver, British Columbia: Mineralogical Association of Canada, 2003. 140 p. (Short Course 32).

VANKO, D.A., BONNIN-MOSBAH, M., PHILLIPOT, P., ROEDDER, E., SUTTON, S.R. Fluid inclusions in quartz from oceanic hydrothermal specimens and the Bingham, Utah porphyry- $\mathrm{Cu}$ deposit: a study with PIXE and SXRF. Chemical Geology, v. 173, p. 227-238, 2001.

VANKO, D.A., MAVROGENES, J.A. Synchrotron-source X-ray fluorescence microprobe: analysis of fluid inclusions. In: MCKIBBEN, M. A., SHANKS III, W. C., RIDLEY, W. I. (eds.). Applications of microanalytical techniques to understanding mineralizing processes. Rev. in Econ. Geol., v. 7, p. 251-263. 1998.

WINICK, H., DONIACH, S. Synchrotron radiation research. New York: Plenum Press, 1980. 754p.

XAVIER, R. P., RADTKE, M., PÉREZ, C. A., SILVA, W. L. The Application of the Micro-SRXRF to Fluid Inclusion Studies with implications to the formation of ore deposits. In: REUNIÃO ANUAL DE USUÁRIOS DO LNLS, 10. Anais... Campinas: Activity Report, Laboratório Nacional de Luz Sincrotron - ABTLus, 1999, p. 1-6.

Artigo recebido em 21/08/2006 e aprovado em 13/09/2006.

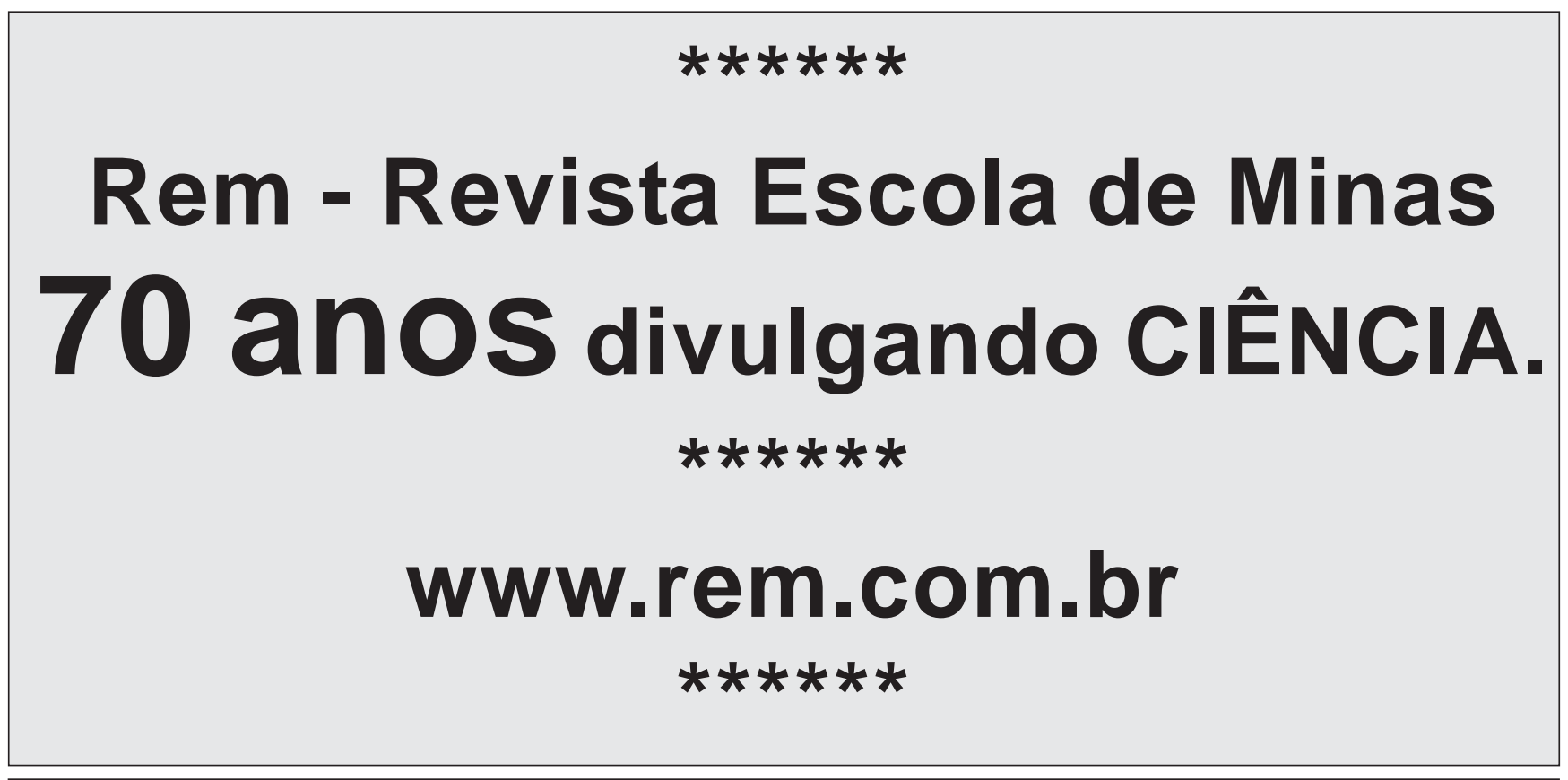

REM: R. Esc. Minas, Ouro Preto, 59(4): 373-377, out. dez. 2006 